\title{
Estimativa da duração do período de molhamento por orvalho em um ecossistema cacau
}

\author{
Hermes A. de Almeida1 \& Regina C. R. Machado ${ }^{2}$
}

\section{RESUMO}

O objetivo deste trabalho foi estimar a duração do período de molhamento por orvalho (DPM), entre 18 e 06 h, utilizando-se dois modelos empíricos: a) o do número de horas com umidade relativa $\geq$ a $97 \%$ (NHUR $\geq 97 \%$ ) e b) o da depressão do ponto de orvalho $\leq 0,5{ }^{\circ} \mathrm{C}$ (NHDPO $\leq 0,5{ }^{\circ} \mathrm{C}$ ). Uma estação meteorológica foi instalada no interior de uma fazenda de cacau, denominada Caprichosa, município de Camacan, BA, Brasil, onde se coletaram dados de temperatura do ar (tar) e de umidade relativa (UR), que foram utilizados nos cálculos das pressões parciais, de saturação vapor de água e temperatura do ponto de orvalho (to). Obteve-se a depressão do ponto de orvalho (DPO) pela diferença entre a to e a tar. Com os valores de UR e DPO se estabeleceram os modelos para as estimativas da DPM, cuja escolha se baseou nos índices estatísticos de precisão, exatidão e confiança. A DPM ocorreu, em média, em mais de $60 \%$ dos dias, embora nos meses de inverno e verão esses percentuais tenham sido contrastantes, isto é, 80,8 e 15,8\%, respectivamente. Os elevados índices estatísticos encontrados permitem estimar, com elevada precisão, a DPM no referido ecossistema cacau. Os modelos são de fácil aplicação e operação, dispensando o uso de sensores e de métodos mais complexos, embora não deva ser aplicado em outras condições e/ou tipos de cultivo sem os ajustes necessários.

Palavras-chave: cacaueiro, microclima, temperatura do ponto de orvalho, umidade relativa do ar

\section{Estimate of the duration of the wetness period by dew in a cocoa ecosystem}

\begin{abstract}
The objective of this study was to estimate the duration of wetness by dew (DWW), from 18 to $06 \mathrm{~h}$, using two empirical models: a) number of hours with relative air humidity $\geq 97 \%$ (NHUR $\geq 97 \%$ ) and b) depression of the dew point $\leq 0.5^{\circ} \mathrm{C}$ $\left(\mathrm{NHDPO} \leq 0.5^{\circ} \mathrm{C}\right)$. A meteorological station was placed in a cocoa plantation at Camacan Bahia, Brazil, in which data were collected for air temperature (tar) and relative humidity (UR). These data were used for calculations of the partial pressure, vapor saturation and temperature of the dew point (to). The depression of the dew point (DWP) was obtained by the difference between to and tar. With the hourly data of UR and DWP the models were established for estimating DWW. The choice of the best model was based on the statistical indexes of precision and accuracy. It was observed that DWW occurred, on average most of the days $(>61 \%)$, although the percentage for winter and summer months was 80.8 and $15.8 \%$ respectively. The high statistical indexes observed here show the viability of the models for estimating, with high precision, the DWW in a cocoa ecosystem. The models are of easy application and operation, dispensing the utilization of sensors and of more complex methods. Howerer, they still need some adjustments to be used for other crops and conditions.
\end{abstract}

Key words: cocoa tree, microclimate, temperature of the dew point air, relative humidity

1 UEPB, CEP 58400-560, Campina Grande, PB. Fone: (83) 3310-7020. E-mail: hermes_almeida@uol.com.br

${ }^{2}$ Centro de Pesquisas do Cacau, CEP 45600-970, Ilhéus, BA. Fone: (73) 3214-7900. E-mail: machareg@terra.com.br 


\section{INTRODUÇÃO}

Molhamento foliar é o termo usado para designar a presença de água na forma líquida, "água livre”, sobre a parte aérea dos vegetais. A maioria dos fungos requer água livre para que ocorra a germinação dos esporos e, por isso, o molhamento por orvalho favorece seu desenvolvimento (Berton \& Melzer, 1984; Butler \& Jadhav, 1991).

O orvalho que ocorre em superfícies naturais, pode ser originado de dois processos distintos: da "precipitação de orvalho" e da "destilação do orvalho”, em que ambos são funções do gradiente de pressão de vapor (Sharma, 1976).

O orvalho se forma como resultado do resfriamento noturno, devido às perdas de radiação de onda longa que reduz a temperatura até atingir o ponto de orvalho, quando ocorre a saturação e, consequentemente, o início do processo de condensação do vapor. Ressalta-se, ainda, que, existindo partículas hidrofílicas no ar, a formação do orvalho pode-se dar muito antes que a umidade relativa atinja $100 \%$, podendo ter início, em média, duas a três horas após o ocaso do sol (Baier, 1996).

O molhamento por orvalho é uma das variáveis ambientais que mais influencia o desenvolvimento de doenças vegetais (Dalla Marta et al., 2005), embora, a duração período de molhamento (DPM) tem sido considerada fator decisivo para desencadear um processo epidemiológico, tais como infecção e esporulação (Sentelhas et al., 2008); no entanto, a DPM não pode ser considerada uma variável unicamente meteorológica porque está relacionada com as propriedades físicas da superfície vegetal e do dossel vegetativo (Madeira et al., 2002). Apesar da importância da duração do molhamento é, na realidade, o binômio temperatura versus DPM que irá condicionar efetivamente o estabelecimento dos patógenos (Pereira et al., 2002).

Destaque-se, ainda, que a variabilidade espacial da DPM tem outras características relacionadas ao microclima das culturas, o que possibilita, portanto, que algumas das partes do dossel vegetativo se encontrem úmidas enquanto outras se apresentem secas (Santos, 2006).

Variáveis meteorológicas, especialmente temperaturas do ar e do ponto de orvalho e umidade relativa constituem, na realidade, uma opção extremamente útil para gerar modelos que possibilitem estimar o período de molhamento por orvalho (Kim et al., 2005; Santos, 2006), razão por que os modelos empíricos podem fornecer boas estimativas da DPM com a vantagem de serem mais econômicos e por dispensar a aquisição e instalação de sensores específicos.

De acordo com Huber \& Gillespie (1992), os modelos para estimar a DPM podem ser agrupados em duas categorias; na dos físicos, que levam em consideração o balanço de energia e na dos empíricos, em que se utilizam os elementos meteorológicos e métodos estatísticos.

Sentelhas et al. (2003) mostraram que a DPM por orvalho na cultura do algodão, obtida por modelo empírico utilizando-se a temperatura do ponto de orvalho e umidade relativa do ar, foi semelhante à encontrada com o modelo físico mais complexo, como o de Penman-Monteith.

Quantificar a DPM por orvalho no ecossistema cacaueiro é muito mais difícil do que a maioria dos outros cultivos, visto que o cacaueiro é cultivado sob a proteção de outras espécies, árvores de sombras e/ou por sistema de plantio em consórcio, o que dificulta ainda mais a sua determinação.

Embora existam diversos equipamentos e/ou métodos de estimativa da duração do período de molhamento, não existe um método específico ou padronizado para quantificar a ocorrência e a duração do orvalho, embora há modelos baseados em uma ou mais variáveis meteorológica, como umidade relativa e temperatura, até modelos mais complexos, os quais usam os princípios do balanço de energia e da resistência aerodinâmica (Madeira et al., 2002).

No entanto, a determinação da DPM se torna necessária para identificar o potencial de riscos de surtos de ocorrência de doenças nas culturas e, portanto, na tomada de decisão quanto à realização dos controles.

Estimar a duração de molhamento por orvalho no ecossistema cacau, utilizando-se os números de horas com umidade relativa, maior ou igual a $97 \%$ (NHUR > 97\%), e os de depressão da temperatura do ponto de orvalho, menor que $<0,5^{\circ} \mathrm{C}\left(\mathrm{DPO}<0,5^{\circ} \mathrm{C}\right)$ foi o objetivo principal deste trabalho.

\section{MATERIAL E MÉTODOS}

O experimento foi conduzido em áreas cultivadas com cacaueiros (Theobroma cacau L), implantados no espaçamento de 3 x 3 m, sob sombreamento de Eritrina (Erythrina glauca Willd), no espaçamento de 24 x 24 m, na Fazenda Caprichosa, localizada no Município de Camacan, BA, Latitude $15^{\circ} 25^{\prime}$ S e Longitude $39^{\circ} 30^{\prime} \mathrm{W}$.

Uma estação meteorológica foi instalada no centro da área experimental e os sensores acerca de $1,7 \mathrm{~m}$ da superfície do solo. No interior do abrigo meteorológico padrão havia um conjunto de termômetros de máxima e mínima, um psicrômetro e um registrador duplo de temperatura e umidade relativa (termohigrógrafo). Computaram-se, dentre os registros contínuos de temperatura do ar (tar) e de umidade relativa (UR), os valores pontuais, a cada hora, ao invés da média horária.

Com esses dados se estimaram, em escala horária e se utilizando a equação de Tetens a pressão de saturação de vapor $\left(e_{s}\right)$ e a pressão parcial de vapor $\left(e_{a}\right)$, em $\mathrm{kPa}$, a temperatura do ponto de orvalho $\left(\mathrm{t}_{\mathrm{o}}\right)$ e a depressão do ponto de orvalho (DPO), em ${ }^{\circ} \mathrm{C}$, mediante as seguintes expressões:

$$
\begin{aligned}
& \mathrm{e}_{\mathrm{s}}(\mathrm{kPa})=0,61 \times 10^{\left[\frac{7,5 \times \operatorname{tar}}{237,3+\operatorname{tar}}\right]} \\
& \mathrm{e}_{\mathrm{a}}(\mathrm{kPa})=\left(\mathrm{e}_{\mathrm{s}} \times \mathrm{UR}\right) \times 0,01 \\
& \mathrm{t}_{\mathrm{o}}\left({ }^{\circ} \mathrm{C}\right)=\frac{237,3 \times \log \left(\mathrm{e}_{\mathrm{a}} / 0,61\right)}{7,5-\log \left(\mathrm{e}_{\mathrm{a}} / 0,61\right)} \\
& \text { DPO }\left({ }^{\circ} \mathrm{C}\right)=\operatorname{tar}-\mathrm{t}_{\mathrm{o}}
\end{aligned}
$$

Estabeleceu-se que o período noturno seria aquele compreendido entre 18:00 e 06:00 h por ser este o intervalo de 
ocorrência natural de orvalho. Utilizando-se a distribuição de frequência contabilizaram-se, para cada hora, dias e mês, as respectivas frações nas quais a umidade relativa e a depressão do ponto de orvalho eram, respectivamente, maior ou igual a $97 \%$ (NHUR $\geq 97 \%$ ) e igual ou menor que $0,5{ }^{\circ} \mathrm{C}$ $\left(\mathrm{DPO} \leq 0,5^{\circ} \mathrm{C}\right)$.

Estimou-se a duração do período de molhamento (DPM) usando-se dois métodos empíricos: o do número de horas com NHUR $\geq 97 \%$ e o da depressão do ponto de orvalho (DPO) com valores iguais ou menores que $0,5^{\circ} \mathrm{C}$. Em observações visuais em condições de campo, constatou-se a presença de orvalho quando os valores da DPO eram $\leq 0,5^{\circ} \mathrm{C}$.

Correlacionando-se os NHUR $\geq 97 \%$ e os $\mathrm{NHDPO} \leq$ $0,5^{\circ} \mathrm{C}$, obteve-se a duração do período de molhamento, em frações de horas, para cada intervalo (das 18 às 06 h).

Os dados estimados pelos dois métodos e pelas respectivas equações de regressão estabelecidas, foram comparados com base nos indicativos estatísticos de precisão (coeficiente de determinação $-\mathrm{R}^{2}$ ), de exatidão (d), expressos pelos índices estatísticos propostos por Willmott et al. (1985), mediante as expressões (5) e de confiança ou desempenho (6), estabelecido por Camargo \& Sentelhas (1997), descritas a seguir:

$$
\begin{aligned}
& \mathrm{d}=1-\left[\frac{\Sigma\left(\mathrm{O}_{\mathrm{i}}-\mathrm{P}_{\mathrm{i}}\right)^{2}}{\Sigma\left(\left|\mathrm{P}_{\mathrm{i}}-\overline{\mathrm{O}}_{\mathrm{i}}\right|+\left|\mathrm{O}_{\mathrm{i}}-\overline{\mathrm{O}}_{\mathrm{i}}\right|\right)^{2}}\right] \\
& \mathrm{c}=\mathrm{d} \sqrt{\mathrm{R}^{2}}
\end{aligned}
$$

Sendo $\mathrm{O}_{\mathrm{i}}$ e $\mathrm{P}_{\mathrm{i}}$ os dados estimados mediantes os métodos e as respectivas equações de regressão estabelecida. A barra do sobrescrito significa a média dos valores estimados pelos métodos.

Todos os cálculos, análises estatísticas e a confecção dos gráficos, foram elaborados utilizando-se a planilha Excel.

\section{RESULTADOS E DISCUSSÃO}

A escolha do período noturno (das 18 às 06 h) se deve única e exclusivamente ao fato do processo de formação do orvalho, seja a precipitação por orvalho ou a destilação por orvalho, suceder com o arrefecimento do ar, até atingir a temperatura do ponto de orvalho, quando então ocorre a condensação.

As observações visuais em condições de campo mostraram que o início da condensação nas partes aéreas do cacaueiro, ocorria mais frequentemente quando a umidade relativa do ar e/ou a depressão do ponto de orvalho eram, respectivamente, $\geq 97 \%$ e $\leq$ a $0,5^{\circ} \mathrm{C}$. Constatou-se que em alguns dias a ocorrência do orvalho em relação a esses valores referenciais ocorria com uma defasagem da ordem de $30 \mathrm{~min}$, resultados que concordam com os encontrados por Sentelhas et al. (2008) em que, segundo eles, diferenças como essas não são significantes, porque medidas de DPM estão sujeitas a erros inerentes à variabilidade espacial do molhamento.

Destaque-se, ainda, neste trabalho que, as temperaturas do ar e umidade relativa foram coletadas a $1,7 \mathrm{~m}$ de altura do solo e não a nível do solo, como as obtidas por Sentelhas et al. (2003); Santos (2006), dentre outros; outra diferença marcante reside na estimativa da DPM, haja vista que esses autores usaram o NHUR $>87 \%$ e DPO $<2,0{ }^{\circ} \mathrm{C}$ e não NHUR $\geq 97 \%$ e DPO $\leq 0,5{ }^{\circ} \mathrm{C}$.

As Figuras $1 \mathrm{~A}$ e $\mathrm{B}$ mostra, respectivamente as médias decimais do número de horas com molhamento por orvalho, durante o período noturno, obtidas a partir do número de hora com umidade relativa $\geq 97 \%$ e da depressão do ponto de orvalho $\leq 0,5^{\circ} \mathrm{C}$, em três situações distintas: média do período e médias para os meses de dezembro e junho.
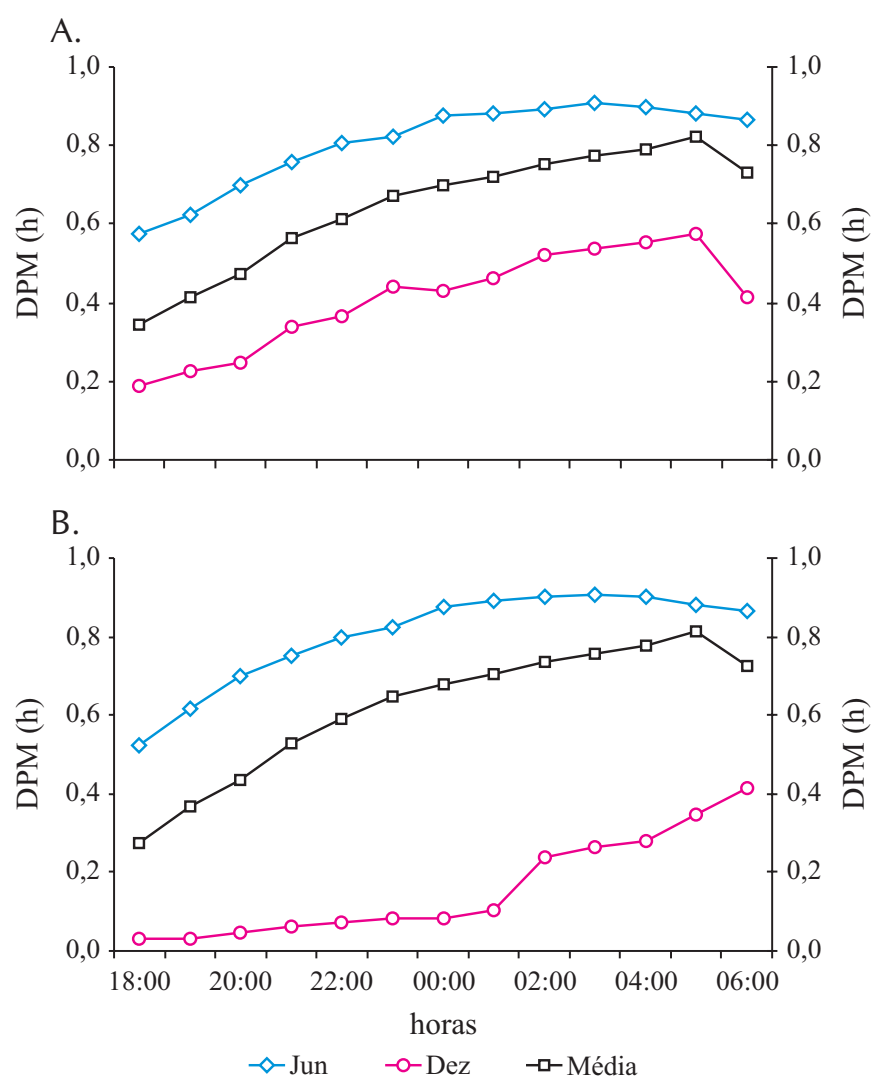

Figura 1. Médias fracionárias do número de horas com molhamento por orvalho, obtidas a partir do NHUR $\geq 97 \%$ (A) e do número de horas com molhamento por orvalho, obtidas a partir do $\mathrm{NHDPO} \leq 0,5{ }^{\circ} \mathrm{C}$ (B); médias de cinco anos

Dos 1724 dias analisados no intervalo entre 18 e $06 \mathrm{~h}$ da manhã (totalizando treze h), houve potencial para formação de orvalho, em média em $64,4 \%$ das h $(8,4$ h), embora em junho este percentual tenha sido de $80,8 \%(10,5 \mathrm{~h})$ contra $40,8 \%$ (5,3 h) em dezembro.

Considerando-se o número de horas diárias com umidade de $90 \%$, constatou-se que este valor ocorreu, em média, em $94,2 \%$ dos dias, sendo que em junho foi de $99,7 \%$ e em dezembro $84,9 \%$, indicando, a priori, o elevado potencial para a ocorrência de molhamento por orvalho na região cacaueira da Bahia.

O uso do método empírico para estimar a DPM com base no número de horas com depressão do ponto de orvalho $\leq 0,5^{\circ} \mathrm{C}$ e não $<2,0^{\circ} \mathrm{C}$, como usado por Gillespie et al. (1993) e Rao et al. (1998), baseou-se nos bons resultados 
encontrados por esses autores ao se comparar seus resultados com os obtidos por modelos físicos mais complexos.

A Figura 1B. apresenta as médias fracionárias do número de horas com molhamento por orvalho durante o período noturno, contabilizadas a partir do número de hora com depressão do ponto de orvalho $\leq 0,5^{\circ} \mathrm{C}$ para três situações distintas: média do período e médias para os meses de dezembro e junho. Observou-se que há variações temporais cujas médias foram, respectivamente, de $61,8 \%$ (8,0 h), 80,3\% $(10,4$ h) e $15,8 \%$ (2 h), embora o número de horas com depressão do ponto de orvalho $\leq 0,5{ }^{\circ} \mathrm{C}$ tenha sido, em junho, cinco vezes maior que em dezembro, ou seja, médias de 10,4 h em junho contra apenas 2,1 h em dezembro.

Fazendo-se uma análise comparativa entre os dois métodos de estimativa da DPM analisados constata-se que os números de horas com DPM foram bem semelhantes, especialmente para as condições médias e para o mês de junho; nota-se, porém, que a DPM média no mês de dezembro (Figura 1B) foi menor que $0,2 \mathrm{~h}$ (12 min), das 18 às $02 \mathrm{~h}$, com um máximo às $06 \mathrm{~h}$ da manhã (com $0,4 \mathrm{~h}=24 \mathrm{~min}$ ), tempos esses que, do ponto de vista prático, podem ser considerados desprezíveis.

Ressalte-se, entretanto, que a não ocorrência de orvalho nas noites do mês de dezembro era prevista, visto que o orvalho pode não se formar, em algumas ocasiões, quando a DPO esteja por volta de $0,5^{\circ} \mathrm{C}$, especialmente durante noites com ventos mais intensos, como as que ocorreram em dezembro em que a formação do orvalho somente ocorreria com DPO a zero grau.

Com relação às diferenças existentes nos números médios de horas com molhamento por orvalho, embora pequena $(0,4 \mathrm{~h})$, acredita-se que essa desigualdade se deva ao método, uma vez que, no da depressão do ponto de orvalho, se utiliza, além de UR, dados derivados de temperatura no cálculo da temperatura do ponto de orvalho.

As Figuras 2A, B e C mostram, respectivamente, as equações de regressão e coeficientes de determinação $\left(r^{2}\right)$ da DPM obtidas em função do NHUR $\geq 97 \%$ e DPO $\leq$ $0,5^{\circ} \mathrm{C}$, no intervalo entre 18 e $06 \mathrm{~h}$. Os indicativos estatísticos mostrados pelos elevados valores dos coeficientes de determinação (de 0,908 a 0,961), revelam a precisão encontrada nos modelos.

Observa-se (Figura 2B), que as médias da DPM, em h, para valores de UR, média inferior a 90\%, foram de no máximo 0,14 h (8,2 min) e aumentaram exponencialmente a partir do valor de UR maior que $90 \%$, cuja duração média foi da ordem de $15 \mathrm{~min}$. Esses pequenos valores podem advir de erros de estimativas que, segundo Sentelhas et al. (2008), podem não ser significante do ponto de vista operacional; já a relação com a DPO (Figura 2C) também segue uma tendência exponencial quase perfeita porém decresce com o aumento da DPO.

Outro aspecto relevante é o padrão de variação da DPM em função da altura da planta; no cafeeiro, por exemplo, o início do período de molhamento ocorre, por último, no topo que nas demais posições e dura uma hora a menos que no interior da folhagem (Santos, 2006).

Resultados encontrados por Rosenberg et al. (1983) mos-

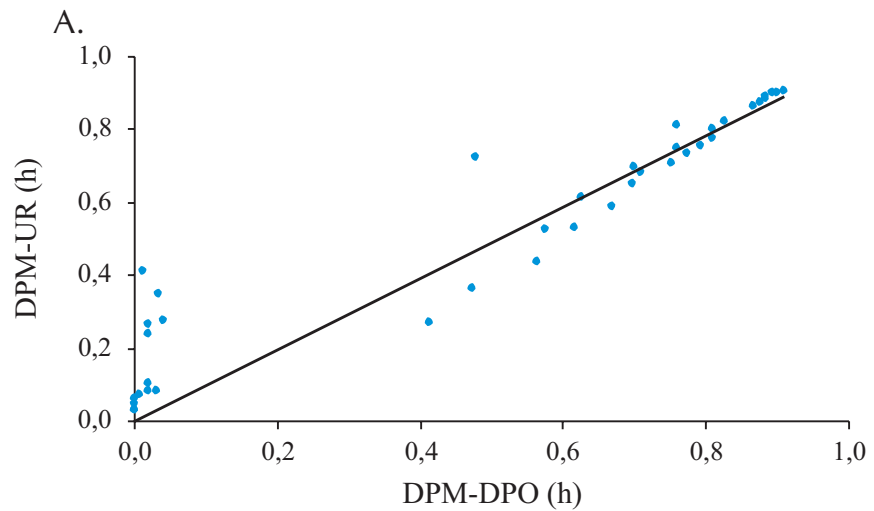

B.
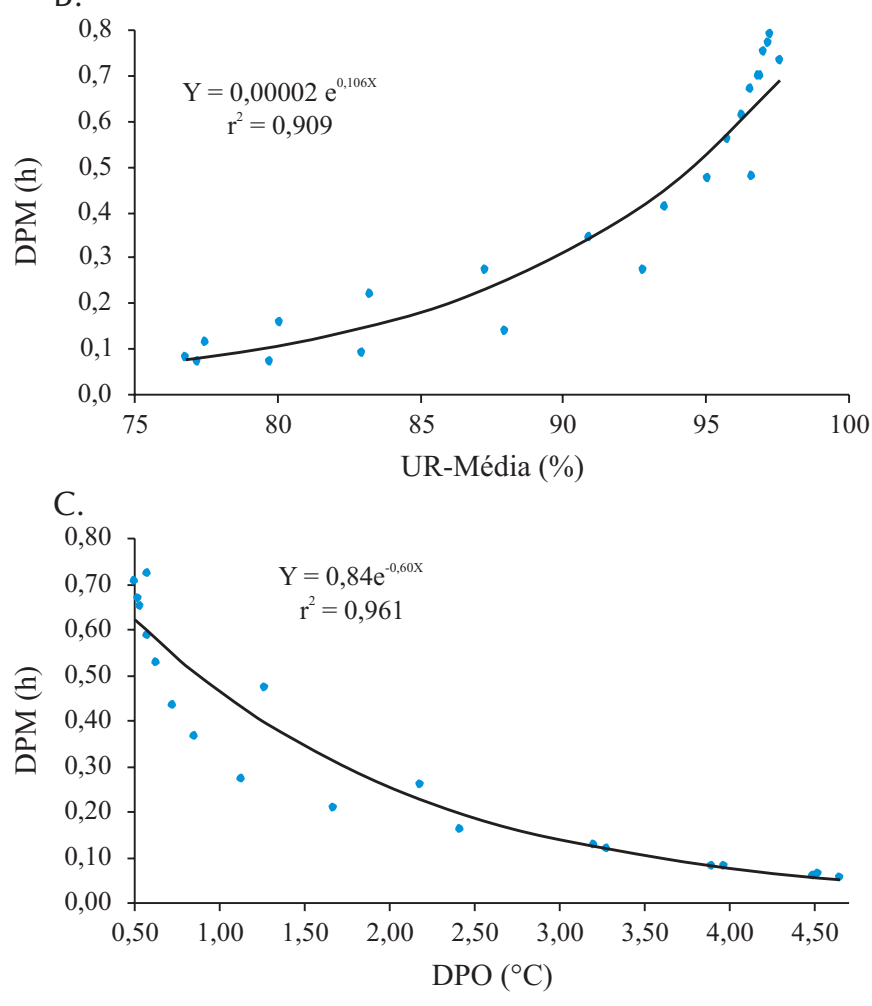

Figura 2. Modelos de regressão da duração de período de molhamento (DPM), para o período noturno, obtidos pelas relações: DPM umidade relativa versus DPM duração do período de molhamento por orvalho (A), da DPM contra umidade relativa média (B) e DPM versus depressão do ponto de orvalho $(C)$, médias de cinco anos

traram que o início de molhamento ocorre mais cedo nas partes mais baixas e no interior da copa das plantas. Para eles, a menor velocidade do vento, nessas posições do dossel, favorece a deposição mais rápida de orvalho sobre a superfície vegetal. Em um ecossistema cacau acredita-se que as partes aéreas abaixo do dossel, por interceptarem mais radiação de ondas longas durante a noite, se resfriam menos e, consequentemente, terão menor tempo para deposição de orvalho.

Comparando a DPM obtida com modelos empíricos e utilizando NHUR $>87 \%$ e DPO $<2,0^{\circ} \mathrm{C}$, Santos (2006) observou que os resultados foram compatíveis com aqueles obtidos por outros autores com modelos mais complexos, embora o modelo empírico tenha apresentado desempenho geralmente melhor para a região para qual ele foi desenvolvido, porém 
tal evento não invalida os modelos, que podem ser ajustados para outras condições; além disso, os citados modelos apresentam, ainda, outras vantagens adicionais, tais como menor número de variáveis de entrada e por serem menos complexos e de fácil interpretação.

Os índices estatísticos de precisão, exatidão e de confiança, são mostrados na Tabela 1 . As pequenas diferenças numéricas entre eles não foram significativas pelo teste t, a 5\% de probabilidade. As avaliações conjuntas dos modelos (precisão e exatidão) mostram índices de confiança (c) superiores a 0,8, considerados ótimos por Camargo \& Sentelhas (1997).

Tabela 1. Índices estatísticos de precisão $\left(\mathrm{R}^{2}\right)$, exatidão (d) e confiança (c) dos modelos para estimar a duração do período de molhamento (DPM), estabelecidos a partir do número de horas com umidade relativa (UR) $\geq 97 \%$ e depressão do ponto de orvalho (DPO) $\leq 0,5{ }^{\circ} \mathrm{C}$, médias de cinco anos

\begin{tabular}{lccc}
\hline Modelos & $\mathbf{R}^{2}$ & $\mathbf{d}$ & $\mathbf{C}$ \\
DPM-UR x DPM-DP0 & 0,908 & 0,999 & 0,943 \\
DPM x URmédia & 0,909 & 0,997 & 0,950 \\
DPM x DP0 & 0,961 & 0,999 & 0,979 \\
\hline
\end{tabular}

Ressalte-se, entretanto, que os índices estatísticos encontrados nos modelos aqui descritos, Tabela 1 , foram bem superiores aos obtidos por Santos (2006) que obteve respectivamente 0,69 e 0,$73 ; 0,87$ e 0,84 e de 0,72 e 0,72 para $^{2}$, d e c nos modelos de NHUR $>87 \%$ e DPO $<2,0^{\circ} \mathrm{C}$, durante a estação seca de julho-outubro de 2005, em Piracicaba, SP.

\section{CONCLUSÕES}

1. A duração do período de molhamento (DPM) por orvalho, estimada pelos métodos do NHUR $\geq 97 \%$ e DPO $\leq$ $0,5^{\circ} \mathrm{C}$, no intervalo entre 18 e $06 \mathrm{~h}$ ocorreu, em média, em 64,4 e $61,7 \%$ dos dias, respectivamente.

2. As médias diárias da DPM nos meses de inverno foram de 80,8 contra $15,8 \%$, nos dias de verão.

3. Os métodos do NHUR $\geq 97 \%$ e $\mathrm{DPO} \leq 0,5^{\circ} \mathrm{C}$ são viáveis para obter estimativas precisas da DPM por orvalho, em ecossistemas cacau, além de fácil utilização e menor custo operacional.

4. As excelentes concordâncias encontradas nos modelos de estimativas da DPM não significam que eles possam ser estendidos a outros cultivos sem os ajustes necessários.

\section{LITERATURA CITADA}

Baier, W. Studies on dew formation under semi-arid conditions. Agricultural Meteorology, v.3, p.103-112, 1996.
Berton, O.; Melzer, R. Controle de sarna da macieira pelo sistema de MILLS. Pesquisa Agropecuária Brasileira, v.19, n.10, p.1211-1217, 1984.

Butler, D. R.; Jadhav, D. R. Requirements of leaf wetness and temperature for infection of groundnut by rust. Plant Pathology, v.40, p.395-400, 1991.

Camargo, A. P.; Sentelhas, P. C. Avaliação do desempenho de diferentes métodos de estimativa da evapotranspiração potencial no estado de São Paulo, Brasil. Revista Brasileira de Agrometeorologia, v.5, n.1, p.89-97, 1997.

Dalla Marta, A.; Magareyb, R. D.; Orlandinia, S. Modeling leaf wetness duration and downy mildew simulation on grapevine in Italy. Agricultural and Forest Meteorology, v.132, n.1-2, p.84-95, 2005.

Gillespie, T. J.; Srivastava, B.; Pitblado, R. E. Using operational weather data to schedule fungicide sprays on tomatoes in Southern Ontario, Canada. Journal of Applied Meteorology, v.32, p.567-573, 1993.

Hubber, L., Gillespie, T. J. Modeling leaf wetness in relation to plant disease epidemiology. Annual Review of Phytopathology, v.30, p.553-577, 1992

Kim, K. S.; Taylor, S. E.; Gleason, M. L.; Villalobos, R.; Arauz, L. F. Estimation of leaf wetness duration using empirical models in northwestern Costa Rica. Agricultural and Forest Meteorology, v.129, n.1-2, p.53-67, 2005.

Madeira, A. C.; Kim, K. S.; Taylor, S. E.; Gleason, M. L. A simple cloud-based energy balance model to estimate dew. Agricultural and Forest Meteorology, v.111, p.55-63, 2002.

Pereira, A. R.; Angelocci, L. R.; Sentelhas, P. C. Agrometeorologia: Fundamentos e aplicações práticas. Guaíba: Agropecuária, 2002. 478p.

Rao, P. S.; Gillespie, T. J.; Schaafsma, A. W. Estimating wetness duration on maize ears from meteorological observations. Canadian Journal of Soil Science, v.78, p.149-154, 1998.

Rosenberg, N. J.; Blad, B. L.; Verma, S. B. Microclimate: The biological environment. New York: John Wiley, 1983, 495p.

Santos, E. A. Duração do período de molhamento foliar: medida com sensores eletrônicos, variabilidade espacial em culturas e estimativas com modelos empíricos. Piracicaba: ESALQ/USP, 2006. 56p. Dissertação Mestrado

Sentelhas, P. C.; Dalla Marta, A.; Orlandini, S.; Santos, E. A.; Gillespie, T. J.; Gleasonc, M. L. Suitability of relative humidity as an estimator of leaf wetness duration. Agricultural and Forest Meteorology, v.148, n.3, p.392-400, 2008.

Sentelhas, P. C.; Gillespie, T. J.; Monteiro, J. E. B. A. Estimating leaf wetness duration on a cotton crop from meteorological data. In: Congresso Brasileiro de Agrometeorologia, 13, 2003, Santa Maria, Anais... Santa Maria: SBA/UFSM, 2003. p.663-664.

Sharma, M. L. Contribution of dew in the hydrological balance of semi-arid grassland. Agricultural Meteorology, v.17, p.321-331, 1976.

Willmott, C. J.; Ackleson, S. G., Davis, R. E; Feddema, J. J.; Klink, K. M.; Legates, D. R.; O’donnell, J.; Rowe, C. M. Statistics for the evaluation and comparison of models. Journal of Geophysical Research, v.90, n.5, p.8995-9005, 1985. 